\title{
IMAGE QUANTIZATION UNDER SPATIAL SMOOTHNESS CONSTRAINTS
}

\author{
Anna Jezierska, Caroline Chaux, Hugues Talbot, and Jean-Christophe Pesquet \\ Université Paris-Est, Lab. Informatique Gaspard Monge, UMR CNRS 8049, \\ Champs-sur-Marne, 77454 Marne-la-Vallée, France \\ \{first.last\}@univ-paris-est.fr
}

\begin{abstract}
Quantization, defined as the act of attributing a finite number of grey-levels to an image, is an essential task in image acquisition and coding. It is also intricately linked to various image analysis tasks, such as denoising and segmentation. In this paper, we investigate quantization combined with regularity constraints, a little-studied area which is of interest, in particular, when quantizing in the presence of noise or other acquisition artifacts. We present an optimization approach to the problem involving a novel two-step, iterative, flexible, joint quantizing-regularization method featuring both convex and combinatorial optimization techniques. We show that when using a small number of grey-levels, our approach can yield better quality images in terms of SNR, with lower entropy, than conventional optimal quantization methods.
\end{abstract}

Index Terms - Convex optimization, combinatorial optimization, proximal methods, graph cuts, image coding, segmentation, denoising, entropy.

\section{INTRODUCTION}

Quantization is a fundamental task in digital image processing. It plays a prominent role in early processing stages such as image digitization, and it is essential in lossy coding. It bears close resemblance to high level tasks such as denoising, segmentation, and data classification. In particular, quantizing a grey scale image in $Q$ levels can be viewed as a classification or segmentation of the image in $Q$ areas following an intensity homogeneity criterion. Each segmented area then corresponds to a decision class of the quantizer.

A classical solution for designing an optimal quantizer of an image is provided by the celebrated Lloyd-Max (LM) algorithm $[1,2]$. An extension to the general vector case is the LBG algorithm [3]. The LM algorithm proceeds iteratively by alternatively optimizing quantization levels and decision levels so as to minimize a flexible quantization error measure. It is known to present good convergence properties in practice. However, one drawback is the lack of spatial regularity of the quantized image. Spatially smooth properties may

THIS WORK WAS SUPPORTED BY THE AGENCE NATIONALE DE LA RECHERCHE UNDER GRANT ANR-09-EMER-004-03. be useful in low-rate compression when using advanced coding algorithms (e.g based on run length, differential or multiresolution techniques). It may also be of interest for quantizing images featuring noise. In the latter case, quantization can be viewed as a means for denoising discrete-valued images that are piecewise constant.

Since the LM algorithm is closely related to K-means, which are widely used in data classification, enforcing spatial smoothness of the quantized image could be achieved by resorting to fuzzy C-means clustering techniques and their extensions [4]. These algorithms are however based on local measures of smoothness.

In this paper, we propose a quantization method that enforces some global spatial smoothness. This is achieved by introducing an adjustable regularization term in the minimization criterion, in addition to a quantization error measure. Similarly to the LM algorithm, the optimal design of the quantizer is performed iteratively by alternating the minimization of a label field $i_{\mathcal{D}}$ and of a quantization level vector $\boldsymbol{r}$. The latter minimization reduces to a convex optimization problem whereas the former is carried out by efficient combinatorial optimization techniques.

The problem is formulated in Section 2 and the notation used throughout the paper is introduced. Section 3 describes the proposed quantizer design algorithm. Section 4 provides more details on the combinatorial optimization step. Finally, some simulation results are provided in Section 5 to show the effectiveness of the proposed quantization method before a conclusion is drawn in Section 6.

\section{PROBLEM}

In order to define a quantizer, we introduce the following variables: $Q$ is a positive integer, $\mathcal{D}=\left(\mathbb{D}_{k}\right)_{1 \leq k \leq Q}$ is a partition of $\{1, \ldots, N\} \times\{1, \ldots, M\}$ and $\boldsymbol{r}=\left(r_{1}, \ldots, r_{Q}\right)$ is vector in $\mathbb{C}_{Q}$, where $\mathbb{C}_{Q}$ is the closed convex cone:

$$
\mathbb{C}_{Q}=\left\{\left(s_{1}, \ldots, s_{Q}\right) \in \mathbb{R}^{Q} \mid s_{1} \leq \cdots \leq s_{Q}\right\} .
$$

The partition $\mathcal{D}$ can be characterized by the label image $\left(i_{\mathcal{D}}(n, m)\right)_{1 \leq n \leq N, 1 \leq m \leq M}$, defined as: for every $(n, m) \in$ $\{1, \ldots, N\} \times\{1, \ldots, M\}$ and $k \in\{1, \ldots, Q\}$,

$$
i_{\mathcal{D}}(n, m)=k \quad \Leftrightarrow \quad(n, m) \in \mathbb{D}_{k} .
$$


A scalar quantized image over $Q$ quantization levels $r_{1}, \ldots, r_{Q}$ associated with the partition $\mathcal{D}$ is then given by

$$
q_{i_{\mathcal{D}, \boldsymbol{r}}}=\left(r_{i_{\mathcal{D}}(n, m)}\right)_{1 \leq n \leq N, 1 \leq m \leq M} \in\left\{r_{1}, \ldots, r_{Q}\right\}^{N \times M}
$$

Let $f=(f(n, m))_{1 \leq n \leq N, 1 \leq m \leq M} \in \mathbb{R}^{N \times M}$ denote the original image. An "optimally" $Q$-level quantized image $q_{i_{\overline{\mathcal{D}}}, \overline{\boldsymbol{r}}}$ of $f$ is usually obtained by looking for $\left(i_{\overline{\mathcal{D}}}, \overline{\boldsymbol{r}}\right) \in$ $\{1, \ldots, Q\}^{N \times M} \times \mathbb{C}_{Q}$ solution to the following problem:

$$
\underset{i_{\mathcal{D}}, \boldsymbol{r}}{\operatorname{minimize}} \varphi\left(q_{i_{\mathcal{D}}, \boldsymbol{r}}, f\right)
$$

where $\varphi$ is some measure of the quantization error. Standard choices for $\varphi$ are:

- the weighted $\ell_{p}$ norm measure $(p \in[1,+\infty[)$

$$
\begin{aligned}
& \left(\forall g=(g(n, m))_{1 \leq n \leq N, 1 \leq m \leq M} \in \mathbb{R}^{N \times M}\right) \\
& \varphi(g, f)=\sum_{n=1}^{N} \sum_{m=1}^{M} \omega_{n, m}|g(n, m)-f(n, m)|^{p}
\end{aligned}
$$

where $\left(\omega_{n, m}\right)_{1 \leq n \leq N, 1 \leq m \leq M} \in\left[0,+\infty\left[{ }^{N \times M}\right.\right.$. As special cases, the mean square error criterion is found when $p=2$, and the mean absolute error criterion when $p=1$.

- the sup norm measure

$$
\begin{aligned}
& \left(\forall g=(g(n, m))_{1 \leq n \leq N, 1 \leq m \leq M} \in \mathbb{R}^{N \times M}\right) \\
& \varphi(g, f)=\max _{(n, m) \in\{1, \ldots, N\} \times\{1, \ldots, M\}}|g(n, m)-f(n, m)| .
\end{aligned}
$$

One of the drawbacks of this approach is that it does not guarantee any spatial homogeneity of the resulting quantized image. To alleviate this shortcoming, we propose to solve the following problem:

$$
\underset{i_{\mathcal{D}}, \boldsymbol{r}}{\operatorname{minimize}} \varphi\left(q_{i_{\mathcal{D}}, \boldsymbol{r}}, f\right)+\rho\left(i_{\mathcal{D}}\right)
$$

where $\rho$ is some regularization function which is used to promote the spatial regularity of the label image. Typical choices for $\rho$ that can be made are the following

- isotropic variation functions

$$
\rho\left(i_{\mathcal{D}}\right)=\mu \sum_{n=1}^{N-1} \sum_{m=1}^{M-1} \psi\left(\left\|\nabla i_{\mathcal{D}}(n, m)\right\|\right), \quad \mu \geq 0
$$

where $\nabla i_{\mathcal{D}}(n, m)=\left(i_{\mathcal{D}}(n+1, m)-i_{\mathcal{D}}(n, m)\right.$, $\left.i_{\mathcal{D}}(n, m+1)-i_{\mathcal{D}}(n, m)\right)$ is the discrete gradient of $i_{\mathcal{D}}$ at location $(n, m)$.

- anisotropic variation functions

$$
\begin{aligned}
& \rho\left(i_{\mathcal{D}}\right)=\mu\left(\sum_{n=1}^{N-1} \sum_{m=1}^{M} \psi\left(\left|i_{\mathcal{D}}(n+1, m)-i_{\mathcal{D}}(n, m)\right|\right)\right. \\
& \left.+\sum_{n=1}^{N} \sum_{m=1}^{M-1} \psi\left(\left|i_{\mathcal{D}}(n, m+1)-i_{\mathcal{D}}(n, m)\right|\right)\right), \quad \mu \geq 0 .
\end{aligned}
$$

In the above two examples, $\psi$ is a function from $[0,+\infty[$ onto $[0,+\infty[$. When $\psi$ is the identity function, the classical isotropic or anistropic total variations are obtained. If $\psi=(\cdot)^{2}$, then a Tikhonov-like regularization is performed. Another interesting choice of $\psi$ is the binary cost function

$$
\left(\forall x \in \left[0,+\infty[) \quad \psi(x)= \begin{cases}0 & \text { if } x=0 \\ 1 & \text { otherwise }\end{cases}\right.\right.
$$

\section{PROPOSED OPTIMIZATION METHOD}

Even if $\varphi(\cdot, f)$ and $\rho$ are convex functions, Problem (2.4) is a nonconvex optimization problem due to the fact that $i_{\mathcal{D}}$ belongs to a nonconvex set of discrete values. In order to solve numerically this problem, we propose to use the following alternating optimization algorithm:

$$
\begin{aligned}
& \text { Fix } Q \in \mathbb{N}^{*} \text { and } \boldsymbol{r}^{(0)} \in \mathbb{C}_{Q} \text {. } \\
& \text { For } \ell=0,1, \ldots \\
& {\left[\begin{array}{l}
i_{\mathcal{D}}^{(\ell)} \in \operatorname{Argmin}_{i_{\mathcal{D}} \in\{1, \ldots, Q\}^{N \times M}} \varphi\left(q_{i_{\mathcal{D}}, \boldsymbol{r}(\ell)}, f\right)+\rho\left(i_{\mathcal{D}}\right) \\
\boldsymbol{r}^{(\ell+1)} \in \operatorname{Argmin}_{\boldsymbol{r} \in \mathbb{C}_{Q}} \varphi\left(q_{i_{\mathcal{D}}^{(\ell)}, \boldsymbol{r}}, f\right)
\end{array}\right.}
\end{aligned}
$$

It is worth noticing that this algorithm constitutes an extension of the LM algorithm which would correspond to the case when $\rho$ is the null function. At each iteration $\ell$, the determination of $i_{\mathcal{D}}^{(\ell)}$ given $\boldsymbol{r}^{(\ell)}$ is a combinatorial optimization problem for which there exist efficient solutions for some choices of $\varphi$ and $\rho$, as shown in the next section.

In turn, if $\varphi(\cdot, f)$ is a convex function, the determination of $\boldsymbol{r}^{(\ell+1)}$ given $i_{\mathcal{D}}^{(\ell)}$ is a conic constrained convex optimization problem the solution of which can be determined numerically. For example, in the case of the weighted $\ell_{p}$-norm criterion, this problem is equivalent to find

$$
\boldsymbol{r}^{(\ell+1)} \in \underset{\boldsymbol{r} \in \mathbb{C}_{Q}}{\operatorname{Argmin}} \sum_{k=1}^{Q} \sum_{(n, m) \in \mathbb{D}_{k}^{(\ell)}} \omega_{n, m}\left|r_{k}-f(n, m)\right|^{p}
$$

It is thus possible to solve (3.1) through existing convex optimization approaches $[5,6]$.

Note that, in the proposed iterative algorithm, it may happen that a decision class $\mathbb{D}_{k_{0}}^{(\ell)}$, for some $k_{0} \in\{1, \ldots, Q\}$ becomes empty at iteration $\ell$. In this case, a random value of

$r_{k_{0}}^{(\ell+1)}$ is drawn, so that $\boldsymbol{r}^{(\ell+1)}$ remains in $\mathbb{C}_{Q}$. It can then be shown that the resulting algorithm has convergence properties similar to the LM algorithm.

\section{OPTIMAL COMBINATORIAL PARTITIONING}

We now consider combinatorial optimization methods for finding

$$
i_{\widehat{\mathcal{D}}} \in \underset{i_{\mathcal{D}} \in\{1, \ldots, Q\}^{N \times M}}{\operatorname{Argmin}} \varphi\left(q_{i_{\mathcal{D}}, \boldsymbol{r}}, f\right)+\rho\left(i_{\mathcal{D}}\right)
$$


for a given value of $\boldsymbol{r} \in \mathbb{C}_{Q}$. Here we seek to use standard methods in combinatorial optimization which have proved useful in applications to denoising. In this context, a common form for regularization problems is the following:

$$
\underset{i_{\mathcal{D}} \in\{1, \ldots, Q\}^{N \times M}}{\operatorname{minimimize}} \phi\left(i_{\mathcal{D}}, f\right)+\rho\left(i_{\mathcal{D}}\right)
$$

where $\phi$ is a data fidelity function, $\rho$ a regularization function, $f$ the initial image and $i_{\mathcal{D}}$ the resulting discrete one. To formulate our problem in this framework, we need to introduce the auxiliary function

$$
\begin{aligned}
\chi_{\boldsymbol{r}}:\{1, \ldots, Q\}^{N \times M} & \mapsto\left\{r_{1}, \ldots, r_{Q}\right\}^{N \times M} \\
i_{\mathcal{D}} & \mapsto q_{i_{\mathcal{D}}, \boldsymbol{r}} .
\end{aligned}
$$

Then, our problem becomes

$$
\underset{i_{\mathcal{D}} \in\{1, \ldots, Q\}^{N \times M}}{\operatorname{minimize}} \varphi\left(\chi_{\boldsymbol{r}}\left(i_{\mathcal{D}}\right), f\right)+\rho\left(i_{\mathcal{D}}\right) .
$$

Note that $\chi_{\boldsymbol{r}}$ is monotonic but nonlinear. Note further that the set $\left\{r_{1}, \ldots, r_{Q}\right\}$ changes at each iteration of the complete algorithm. However, during the regularization step this set is fixed, so we are free to choose any optimization method that can be framed as in (4.2).

In this article we use graph cut-based algorithms, which have been shown to be effective in the context of smoothing, denoising and segmentation [7]. More specifically, a solution can be found at each regularization step using the graph proposed by Ishikawa [8], with some small modifications. Namely, i) labels $\iota$ take values from 1 to $Q$, ii) data edges for each vertex $u_{\iota, j}$ have capacity $\varphi\left(r_{\iota}, f\left(n_{j}, m_{j}\right)\right)$, and iii) penalty edges have capacity $\mu$. In case of few quantized levels $Q$, the Ishikawa framework is very efficient. This graph method is known to yield globally optimal solutions for convex functions $\varphi$ and $\rho$ as in (2.6) with $\psi$ the identity function. Alternatively, the convenient though suboptimal alphaexpansion algorithm [7] can be used for all combinations of $\varphi$ and $\psi$, where $\rho$ is the anisotropic TV of (2.6), as described in Section 2. In order to solve Problem (4.3) with the $\alpha$ expansion algorithm, we propose to define the capacities of edges as described in Table 1 . Note that $\alpha$ takes values from 1 to $Q$.

Other optimization methods can also be used. When minimizing isotropic TV as in (2.5), one might want to use for instance Chambolle's algorithm [9]. Similarly to the Ishikawa framework, we can obtain the global optimum in this case. In the following, due to lack of space, we illustrate our proposed method only using graph-cut based regularization methods.

\section{SIMULATION EXAMPLES}

In this section, we present two experiments in order to demonstrate the practical performance of our method in case of: i) low resolution quantization, and ii) quantization in the presence of noise.
Table 1: Capacity for the $\alpha$-expansion graph $\left(\left[x_{1}, x_{2}\right]\right.$ denotes an edge between nodes $x_{1}$ and $x_{2}, i_{\mathcal{D}}\left(n_{u}, m_{u}\right)$ is denoted by $i_{u}$, $f\left(n_{u}, m_{u}\right)$ is denoted by $f_{u},\{u, v\}$ denotes a pair of neighbouring nodes $u$ and $v$ ).

\begin{tabular}{|l|l|l|}
\hline edge & capacity & condition \\
\hline$[\bar{\alpha}, u]$ & $+\infty$ & $\forall u, i_{u}=\alpha$ \\
{$[\bar{\alpha}, u]$} & $\varphi\left(r_{i_{u}}, f_{u}\right)$ & $\forall u, i_{u} \neq \alpha$ \\
{$[\alpha, u]$} & $\varphi\left(r_{\alpha}, f_{u}\right)$ & $\forall u$ \\
{$\left[u, t_{\{u, v\}}\right]$} & $\psi\left(\left|i_{u}-\alpha\right|\right)$ & \\
{$\left[t_{\{u, v\}}, v\right]$} & $\psi\left(\left|\alpha-i_{u}\right|\right)$ & $\forall\{u, v\}, i_{u} \neq i_{v}$ \\
{$\left[\bar{\alpha}, t_{\{u, v\}}\right]$} & $\psi\left(\left|i_{u}-i_{v}\right|\right)$ & \\
{$[u, v]$} & $\psi\left(\left|i_{u}-\alpha\right|\right)$ & $\forall\{u, v\}, i_{u}=i_{v}$ \\
\hline
\end{tabular}

We compare our approach with the LM method [2]. The algorithm performance is measured by the SNR between the original and quantized images and also by the the Shannon entropy of order $(2,2)$ (that is the entropy over image blocks of size $3 \times 3$ ). In the following experiments, we used 8 bit microscopy images of size $512 \times 512$ from public domain (source: http://remf.dartmouth.edu).

First, we consider grey-scale image quantization over 8 levels. The original image is shown in Fig.1(a). Here we minimize the energy function defined by (2.4), with function $\varphi$ defined as the $\ell_{2}$ norm and $\rho$ defined by (2.6) where $\psi$ is the identity. The regularization parameter $\mu$ was hand-optimized to 400. The global optimum solution to the problem can be found with the modified Ishikawa graph shown in Section 4. Both methods, LM and ours, were initialized with uniform decision levels. In order to solve (3.1), the FISTA algorithm [10] was used. As expected, Fig.1 shows that the images have a better spatial smoothness with our method than with LM. This is also verified by inspecting the entropy value, which in our case is equal to $0.56 \mathrm{bpp}$ and in case of LM to $0.84 \mathrm{bpp}$. In this example, we have shown that, in case of quantization with high reduction of levels, our method provides smaller entropy rate while maintaining the desired fidelity.

Next, we consider the problem of grey-scale image quantization over 32 levels in the presence of noise. To generate a noisy image (Fig. 2(b)) from the original one (Fig. 2(a)), we added a zero-mean Laplacian noise with variance 202 (this corresponds to an initial SNR between original and noisy image equal to $18 \mathrm{~dB}$ ). We applied our algorithm minimizing energy (2.4), where $\varphi$ is the $\ell_{1}$ norm, and $\rho$ is defined by (2.6) where $\psi$ is the binary cost-function (2.7). The associated regularization parameter $\mu$ was experimentally chosen to 15 . Both methods, LM and ours, were initialized with decision levels computed on a cumulative histogram. To minimize the energy, we used a modified alpha-expansion graph as described in Section 4. One can observe that there is almost no noise in our result (Fig. 2(d)), while the LM (Fig. 2(c)) pre- 


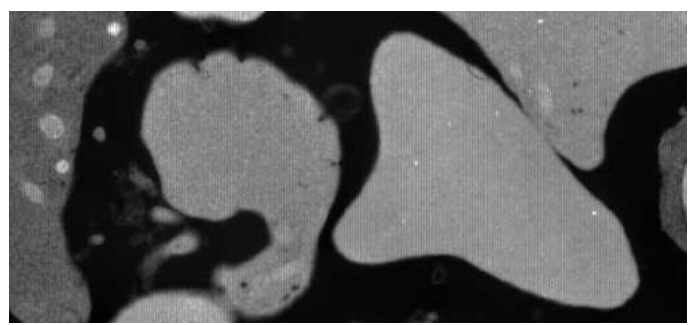

(a)

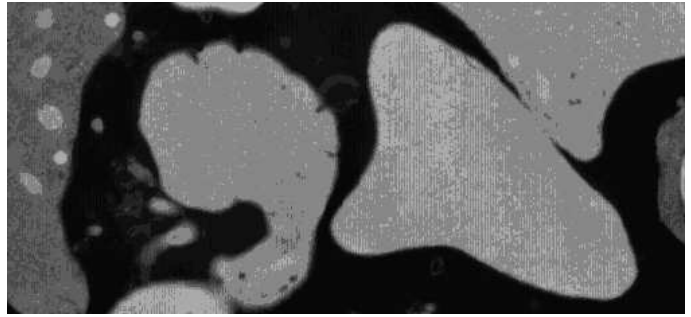

(b)



(c)

Fig. 1: Figures $(a, b, c)$ illustrate fragment of original image, LM and our result, respectively.

serves noise in the image. This is also verified by inspecting SNR values, which are equal to $27.6 \mathrm{~dB}$ for our method and $18.3 \mathrm{~dB}$ for LM. The difference is even greater in terms of entropy: we obtained $0.49 \mathrm{bpp}$ for our method and $1.64 \mathrm{bpp}$ for LM. In this example, we have shown, that in case of quantization in the presence of noise, our method reconstructs the original image, while quantization is being performed.

\section{CONCLUSION}

In this paper, we have proposed a new quantization approach while enforcing spatial smoothness in the resulting image. We have shown that this approach is robust to the presence of noise. These features may be interesting in the context of image compression, in particular to avoid sparing bitrate in coding noise corrupting the image in some applications. We have shown that our method may outperform standard quantization design methods, both in terms of entropy and visual quality.

In future work, we will extend our work to multichannel images and vector quantization.

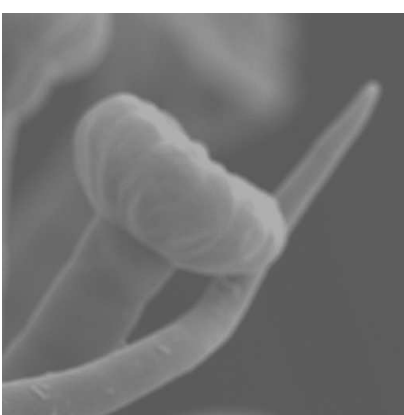

(a)

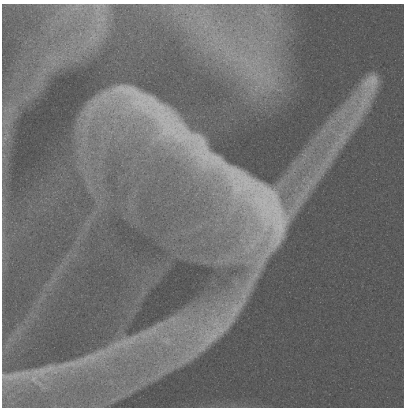

(c)

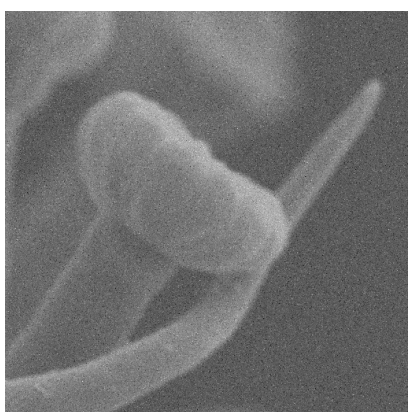

(b)

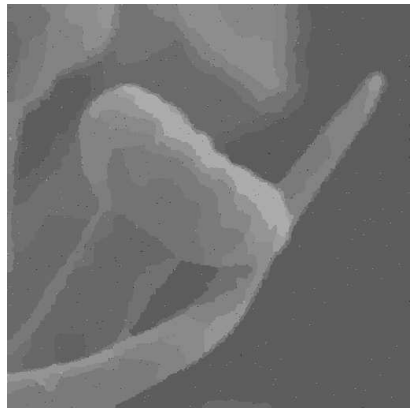

(d)
Fig. 2: Figures $(a, b)$ illustrate original and noisy images, respectively. Images after quantization are presented in Figure (c) for LM algorithm and (d) for our method.

\section{REFERENCES}

[1] J. Max, "Quantizing for minimum distortion," IRE Trans. on Inform. Theory, vol. 6, no. 1, pp. 7-12, Mar. 1960.

[2] S. Lloyd, "Least squares quantization in PCM," IEEE Trans. Inform. Theory, vol. 28, no. 2, pp. 129-137, Mar. 1982.

[3] Y. Linde, A. Buzo, and R. Gray, "An algorithm for vector quantizer design,” IEEE Trans. Commun., vol. 28, no. 1, pp. 84-95, Jan. 1980.

[4] K. Chuang, H. Tzeng, S. Chen, J. Wu, and T. Chen, "Fuzzy c-means clustering with spatial information for image segmentation," Comput. Med. Imag. Graph., vol. 30, pp. 9-15, 2006.

[5] S. Boyd and L. Vandenberghe, Convex Optimization, Cambridge University Press, Cambridge, England, Mar. 2004.

[6] P. L. Combettes and J.-C. Pesquet, "Proximal splitting methods in signal processing," in Fixed-Point Algorithms for Inverse Problems in Science and Engineering, H. H. Bauschke, R. Burachik, P. L. Combettes, V. Elser, D. R. Luke, and H. Wolkowicz, Eds. Springer-Verlag, New York, 2010.

[7] Y. Boykov, O. Veksler, and R. Zabih, "Fast approximate energy minimization via graph cuts," IEEE Trans. Pattern Anal. Mach. Int., vol. 23, no. 11, pp. 1222-1239, Nov. 2001.

[8] H. Ishikawa and D. Geiger, "Mapping image restoration to a graph problem," in IEEE-EURASIP Workshop Nonlinear Signal Image Process., Antalya, Turkey, Jun. 20-23 1999, pp. 189-193.

[9] A. Chambolle, "An algorithm for total variation minimization and applications," J. Math. Imaging Vis., vol. 20, no. 1-2, pp. 89-97, 2004.

[10] A. Beck and M. Teboulle, "A fast iterative shrinkage-thresholding algorithm for linear inverse problem," SIAM J. Imaging Sciences, vol. 2, pp. 183-202, 2009. 